Jap. J. Physiol., 28, 737-747, 1978

\title{
Excitatory and Inhibitory Electrodermal Reflexes Evoked by Cutaneous Stimulation in Acute Spinal Cats
}

\author{
Kenichi Ito, Masakazu Kaseda, Akio Sato, \\ and Yuko Torigata \\ Department of Physiology, Tokyo Metropolitan Institute of Gerontology, \\ Sakae-cho, Itabashi-ku, Tokyo, 173 Japan
}

\begin{abstract}
The electrodermal reflex (EDR) was recorded from the paws of acute spinal cats by a DC potential recording method. The EDR was produced either by pinching the skin in various areas of the trunk, or by electrical stimulation of cutaneous afferents of various spinal segments. It was found that not only excitatory but also inhibitory EDRs were produced and that occurrence of the excitatory and inhibitory EDRs depended on both the segmental position and the laterality of the stimulated area. Cutaneous stimulation generally produced excitatory EDRs more frequently than inhibitory ones, except when afferent inputs en ered the spinal cord at or close to the segments of sudomotor outflow; in the latter case inhibitory EDRs were comparatively frequent and strong. Maximal inhibitory EDRs were elicited by stimulation of the group II afferent fibers of the cutaneous nerves only, whereas maximal excitatory EDRs were elicited after stimulation of the group II, III and IV afferent fibers of cutaneous origin.
\end{abstract}

Activity of the sweat glands produces changes in potentials or in impedances of the skin (WANG, 1957, 1958). Reflex responses of skin potential or impedance produced by stimulation are called "galvanic skin reflex" (GSR) (GILDEMEISTER, 1928; WANG, 1957, 1958) or "electrodermal reflex" (EDR) (WANG, 1964). In this paper the term "EDR" will be employed.

In the cat, it is known that the EDR consists of simple monophasic waves which are easily analyzed and many studies of the central mechanisms of the sympathetic nervous system have been made using the EDR as an indicator. Among them, the studies of Wang and his colleagues are most extensive. Their conclusions (WANG, 1964) about the neural control mechanisms of the EDR can be summarized as follows: (1) only excitatory EDRs are produced at the spinal level, and (2) the spinal excitatory center for the EDR is controlled by excitatory and inhibitory centers in the brain. Wang's observations have been confirmed by many investigators.

Received for publication February 10, 1978

伊藤憲一, 加世田正和, 佐藤昭夫, 鳥潟裕子 
However, the observations of Wang and his co-workers were limited to EDRs elicited from limb afferent nerves with no attention being paid to those elicited from trunk afferent nerves. It would thus seem that Wang's conclusion regarding the central nervous control of EDRs should not be generalized.

In the present experiments, attempts were made in acute spinal cats to elicit EDRs by stimulating the skin and cutaneous afferent nerves in the trunk. We observed both inhibitory and excitatory EDRs, and found that the EDR was either inhibitory or excitatory according to the level of the spinal segment stimulated and the laterality of the stimulation to the recording site.

\section{METHODS}

Experiments were performed on 51 cats weighing $2.2-4.6 \mathrm{~kg}$. They were anesthetized with ether and the spinal cord was transected at C1-2. Immediately after spinalization, ether anesthesia was discontinued and respiration was maintained by a respirator (Acoma-100) through a tracheal cannula. End-expiratory $\mathrm{CO}_{2}$ was kept between 2.5 and $3.0 \%$ (Beckman $\mathrm{CO}_{2}$ analyzer LB-1). In most cases gallamine triethiodide (Teisan, Teikoku Chemicals) was given for immobilization.

The vagus nerve was severed bilaterally at the cervical level to prevent bradycardia, and $5-10 \mathrm{ml}$ of a $6 \%$ solution of dextran (Macrodex-D, AB Pharmacia) was injected into the jugular vein through a catheter to maintain the systolic blood pressure (measured through a catheter in the common carotid artery) above $90 \mathrm{mmHg}$. Rectal temperature was measured with a thermistor thermometer which in turn controlled an infrared lamp and an electric heating pad to heat the animal. In this way the animal's rectal temperature was maintained at $38.0 \pm$ $0.5^{\circ} \mathrm{C}$. Room temperature was kept at $26 \pm 2{ }^{\circ} \mathrm{C}$.

The EDRs were recorded from the central pad of each of the four paws using a zinc plate electrode (about $1 \mathrm{~cm}^{2}$ ) with an indifferent electrode placed on a shaved skin area about $3 \mathrm{~cm}$ proximal to the paw. A mixture of zinc sulfate solution and kaoline paste was used to secure contact between the electrode and the skin. The skin potentials were fed into a DC amplifier whose output was continuously recorded on paper. In some cases the EDRs were averaged by a signal analyzer (HP 5480B).

In 36 cats EDRs were produced by stimulating the skin mechanically in many parts of the trunk. To define the locations of skin stimulation in these cases, the surface of the trunk was divided into 16 longitudinal columns and each column was further divided into small quadrangles by transverse lines which were drawn so as to replicate the dermatomes. The hair was removed with depilatory ointment (Shiseido). Stimulation was made by pinching about $1 \mathrm{~cm}^{2}$ of the skin with a pair of forceps with a force of about $3 \mathrm{~kg}$. In 15 cats, the EDRs were produced by stimulating afferent nerves electrically. For this purpose the skin was cut along the dorsal medline between the T6 and L4 spinal levels, and the 
lateral branches of the cutaneous nerves of the trunk (ramus cutaneus lateralis) were isolated and cut close to the skin. In each experiment at least 3 cutaneous nerves of different spinal segments were dissected. The proximal end of a severed nerve branch was put on a bipolar electrode and stimulated with electrical square pulses (Digitimer type 3290, 2521, 2533). The types of afferent fibers stimulated were identified by monitoring compound action potentials $3-4 \mathrm{~cm}$ proximal to the stimulus site. Nerve branches were kept in a pool of warm liquid paraffin.

\section{RESULTS}

Experiments began about $2 \mathrm{hr}$ after spinal transection and cessation of ether anesthesia. The paws usually showed non-synchronized, spontaneous potential waves (SPWs) of negative polarity, as LADPLI and WANG first found in 1960. We confirmed that these SPWs were completely abolished either by i.v. administration of $0.15 \mathrm{mg} / \mathrm{kg}$ atropine (cholinergic blocker) or by cutting the sciatic and brachial nerves. The SPWs were about $1 \mathrm{mV}$ in amplitude and the frequencies varied from 10 to $35 / \mathrm{min}$. Both amplitude and frequency of the SPWs gradually declined during the experiments which lasted for 8 to $10 \mathrm{hr}$. The SPWs could be increased in amplitude and made to appear more frequently for about $30 \mathrm{~min}$ by tactile stimulation of the perineal skin for 30 to $60 \mathrm{sec}$. This enabled maintenance of stable SPWs during long experiments.

\section{Excitatory and inhibitory EDRs produced by pinching the skin of the trunk}

Figure 1 demonstrates that pinching the skin of the abdomen and chest for $6 \mathrm{sec}$ produced an upward (negative) and a downward (positive) deflection of the baseline, respectively, in the potential record of the forepaw. The reflex responses

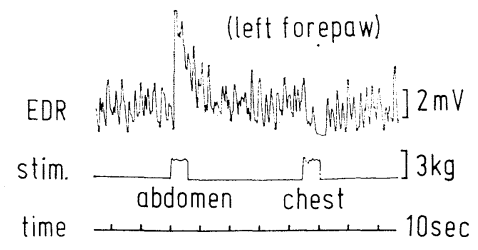

Fig. 1. Excitatory and inhibitory EDRs recorded from left forepaw. Pinching of the skin for $6 \mathrm{sec}$ was made in the abdominal and chest regions as indicated in the middle trace. Time: $10 \mathrm{sec}$ markers. Negativity of the EDR is indicated upward in this and all subsequent records.

which appear as baseline shifts in the negative direction or those in which the SPW frequency increases without changes in the baseline (not shown) are referred to as excitatory EDRs. Conversely, reflex responses in which the baseline shifts in the positive direction or those in which the SPW frequency decreases without changes in the baseline (not shown) are referred to as inhibitory. EDRs. Oc- 
casionally inhibitory EDRs were found to be preceded by small, transient excitatory EDRs lasting only 1-2 sec (not shown). Such EDRs of mixed type were so infrequent and inconsistent that the conditions of their appearance were not analyzed in the present experiment. These mixed EDRs were, however, classified as inhibitory for convenience. Both the excitatory and inhibitory EDRs were abolished either by cutting the sciatic and brachial nerves or by i.v. injection of atropine $(0.15 \mathrm{mg} / \mathrm{kg})$.

As shown in Fig. 2, pinching the skin at different regions on the ventral and dorsal midlines produced excitatory $(\mathrm{C}, \mathrm{D}, \mathrm{F}, \mathrm{G}, \mathrm{H}, \mathrm{J}, \mathrm{K}, \mathrm{M}, \mathrm{O}, \mathrm{P}, \mathrm{Q})$ or inhibitory $(\mathrm{B}, \mathrm{E}, \mathrm{L}, \mathrm{N})$ EDRs in the left forepaw and hindpaw. The excitatory EDR usually started $0.5-0.7 \mathrm{sec}$ after the onset of pinching, reached a maximum within $1-3 \mathrm{sec}$ and gradually decayed within $5-15 \mathrm{sec}$. The inhibitory EDR started $0.5-$ $3.0 \mathrm{sec}$ after the onset of pinching and reached a maximum within $2-7 \mathrm{sec}$. It required $2-15 \mathrm{sec}$ for the inhibitory EDR to disappear completely.
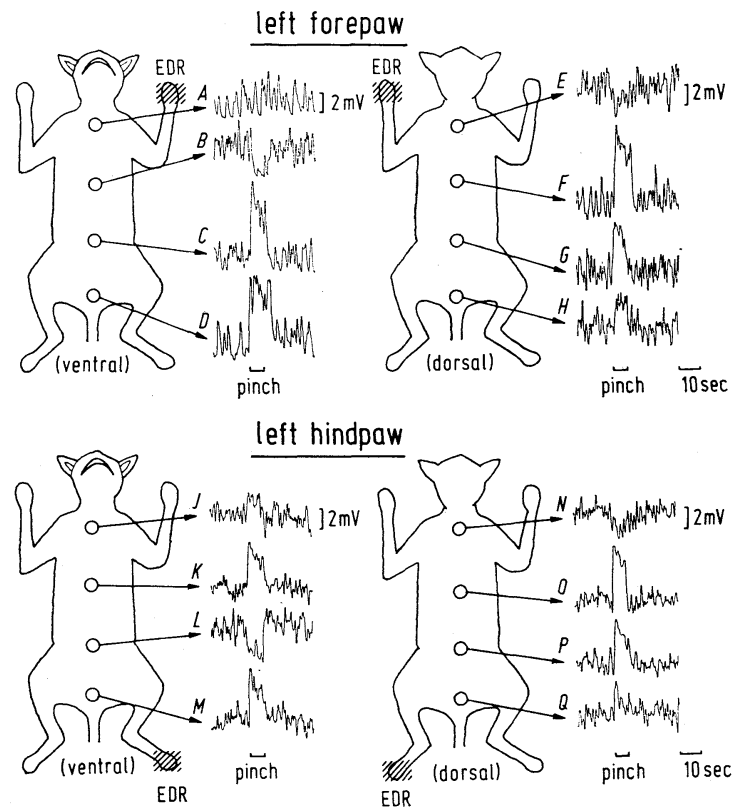

Fig. 2. EDRs produced by pinching skin on ventral and dorsal midlines in an acute spinal cat. A-H, EDRs recorded from left forepaw; J-Q, EDRs recorded from left hindpaw. The skin was pinched for $6 \mathrm{sec}$ as indicated by a horizontal bar below each column of records.

Figure 3 is a summary of all the observed EDRs, both excitatory and inhibitory, of the left forepaws and the left hindpaws of 27 animals, as a function of the stimulus site. Usually, at each stimulus site an area of about $1 \mathrm{~cm}^{2}$ was pinched for $6 \mathrm{sec}$ three times at about $30 \mathrm{sec}$ intervals. By taking the mean of the three 
location and frequency of EDRs
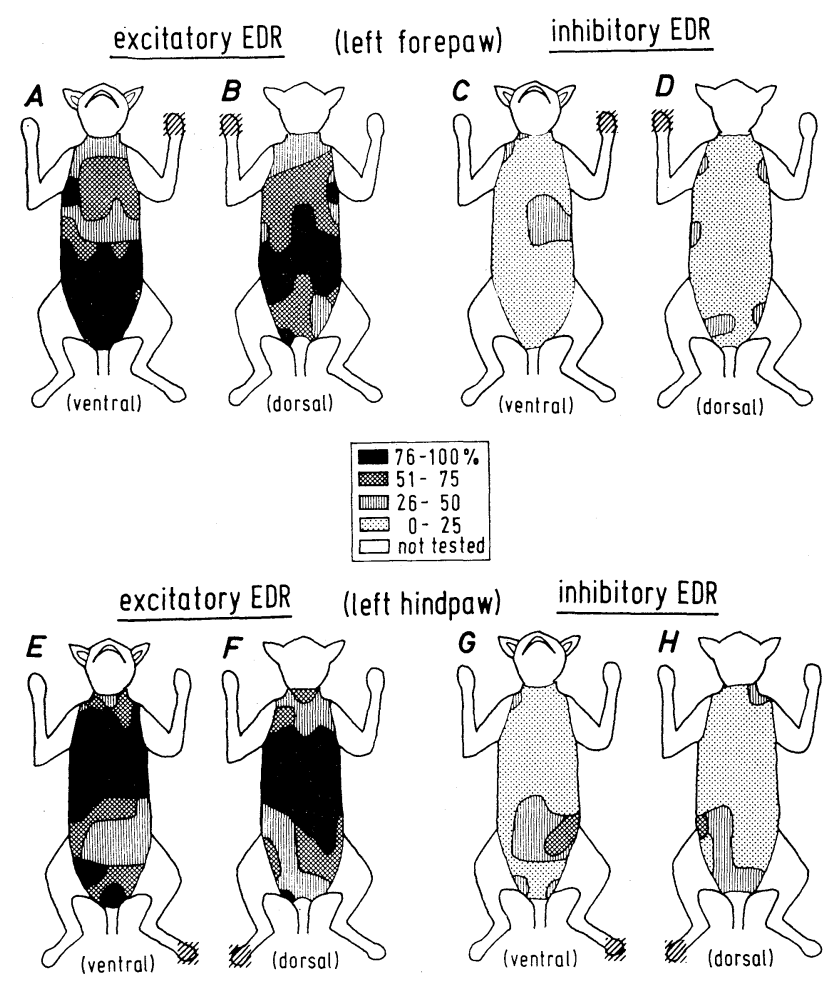

Fig. 3. Frequencies of occurrence of excitatory and inhibitory EDRs as a function of the stimulus site. EDRs were recorded in 27 animals from the left forepaw and hindpaw by pinching the skin at different sites of the surface of the trunk. For each stimulus site the frequencies of occurrence of the excitatory and inhibitory EDRs were calculated in percentages of the total number of animals tested. On the figures of animal's body they are indicated with different hatches as shown in the inset key. A-D, EDRs of left forepaw; E-H, EDRs of hindpaw. A, B, E, F, excitatory EDRs; C, D, G, H, inhibitory EDRs.

EDRs thus recorded, each stimulus site was classified as either excitatory, inhibitory or non-effective for each cat. After a total of 27 animals were thus examined, each stimulus site was classified according to the frequencies of occurrence for both types of response. The frequencies of occurrence, expressed as percentages of the total number of animals, are shown in Fig. 3 with different hatchings for 4 groups of $0-25,26-50,51-75$ and $76-100 \%$. The frequencies of occurrence of the excitatory EDR are illustrated to the left and those of the inhibitory EDR to the right.

The EDR of the forepaw. Excitatory EDRs were produced more frequently from the caudal thoracic, lumbar and sacral skin than from the cervical or rostral 
and mid-thoracic skin (Fig. 3A and B). Inhibitory EDRs were produced much less frequently than excitatory ones (Fig. 3C and D).

The EDR of the hindpaw. Excitatory EDRs were produced most frequently from the rostral and mid-thoracic skin (Fig. 3E and F). Stimulation of the caudal thoracic and lumbar skin elicited inhibitory EDRs (Fig. 3G and $\mathrm{H}$ ).

Laterality. Excitatory EDRs were produced more often by stimulating the skin area contralateral to the paw being observed (Fig. 3A, B, E and F), whereas there was a tendency for inhibitory EDRs to result from ipsilateral stimulation (Fig. 3C, G and $\mathrm{H}$ ).

2. Excitatory and inhibitory EDRs produced by electrical stimulation of cutaneous afferent nerves

Figure 4 shows EDRs recorded simultaneously from the left forepaw and the right hindpaw in one spinal animal by electrical stimulation of the right lateral cutaneous nerves of the T8, T10, T13, L2 and L3 spinal segments. In this case

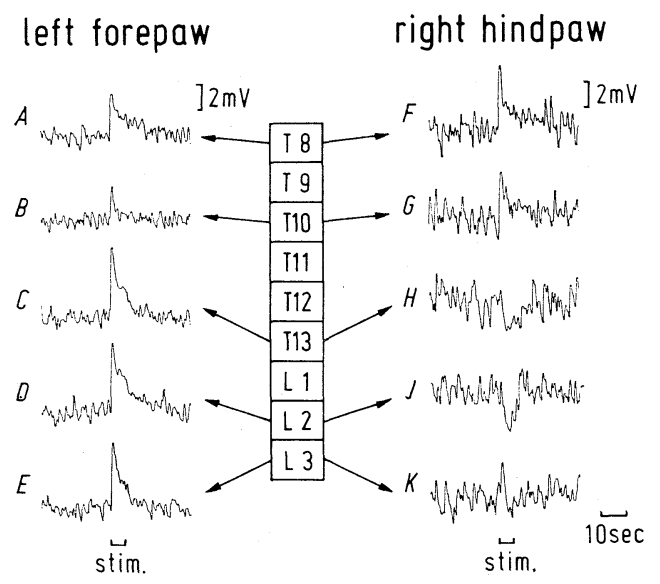

Fig. 4. EDRs produced by electrical stimulation of cutaneous afferent nerves at indicated spinal levels in one acute spinal cat. A-E, EDRs recorded from left forepaw; F-K, EDRs recorded from right hindpaw. Right lateral cutaneous branches of the T8 $(\mathrm{A}, \mathrm{F})$, T10 (B, G), T13 (C, H), L2 (D, J) or L3 (E, K) spinal segments were stimulated. Parameters of stimulation: intensity, $20 \mathrm{~V}$; frequency, $20 \mathrm{~Hz}$; pulse duration, $1 \mathrm{msec}$; stimulus duration, $6 \mathrm{sec}$. Stimulus marker is given as a horizontal bar below each column of records.

the stimulation had the following parameters: intensity, $20 \mathrm{~V}$; pulse duration, $1 \mathrm{msec}$; frequency, $20 \mathrm{~Hz}$; stimulus duration, $6 \mathrm{sec}$. Twenty volts was supramaximal for all nerve fibers (i.e., groups II-IV). As can be seen, the excitatory (A-G and $\mathrm{K}$ ) and inhibitory ( $\mathrm{H}$ and $\mathrm{J}$ ) EDRs were similar to those produced by pinching the skin. It is noteworthy that the EDR recorded from the hindpaw was either excitatory or inhibitory depending on the segmental level stimulated. 
Though the EDRs recorded from the forepaw were all excitatory in this particular case, their magnitude was also a function of the segmental level stimulated. Of special interest is the fact that stimulation of the cutaneous afferent nerves at the T13 and L2 levels produced opposite effects depending on the recording site; an excitatory effect on the left forepaw and an inhibitory effect on the right hindpaw.

In total, 10 spinal cats were studied by stimulating the cutaneous nerves of the T6 to L4 segments and recording EDRs from 4 paws. The effect on a given recording site exerted by each nerve was classified as either excitatory, inhibitory or non-responsive from at least three stimulation trials in each cat. The results are summarized in Fig. 5. The frequencies of occurrence of excitatory

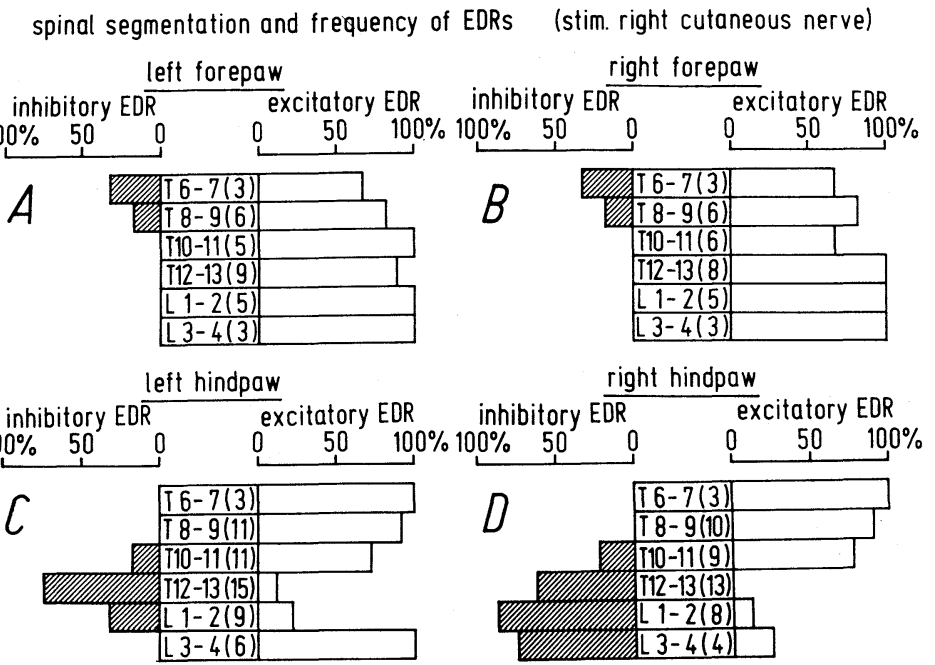

Fig. 5. Frequencies of occurrence for excitatory and inhibitory EDRs as a function of the segmental level stimulated. EDRs were recorded in 10 cats from the left forepaw (A), right forepaw (B), left hindpaw (C) and right hindpaw (D). Right cutaneous nerve branches of the T6 to L4 spinal segments were stimulated. For each pair of the spinal segments, frequencies of occurrence were expressed as percentages of the total number of the nerves tested. They are shown as histograms with white columns for excitatory EDRs and with hatched columns for inhibitory EDRs. Percentages for "non-responsive" are not shown. Parenthesized figures in the frequency histograms are numbers of nerves tested.

(white column) and inhibitory (hatched column) EDRs are given in percentages of the total number of nerves tested. The numbers in parentheses are the numbers of nerves stimulated in the 2 segments. In this series of experiments the intensity of electrical stimulation was supramaximal for all cutaneous nerve fiber groups (II to IV) with a frequency of $20 \mathrm{~Hz}$ and stimulus duration of $6 \mathrm{sec}$.

The EDR of the forepaw. The excitatory. EDR was produced with greater 
frequency and magnitude by stimulation of cutaneous nerves of the T6 to L4 spinal segments, independent of the laterality of stimulation. The inhibitory EDR was much less frequently observed and, when present, it was elicited mostly by activation of the T6-T9 afferents.

The EDR of the hindpaw. The excitatory EDR in both the ipsi- and contralateral hindpaws was more frequently produced than the inhibitory one by stimulation of cutaneous nerves of the T6-T11 spinal segments. Excitatory EDRs were also frequently produced by stimulation of the L3-L4 spinal segments in the contralateral hindpaw, but not in the ipsilateral hindpaw. On the other hand, the inhibitory EDR was more frequently produced than the excitatory one by stimulation of the T12-L2 spinal segments in both the ipsi- and contralateral hindpaws. In addition, inhibitory EDRs in the ipsilateral hindpaw were also frequently produced by stimulation of the L3-L4 spinal segment.

3. Afferent fiber groups of the cutaneous nerve producing the EDR

The data in Fig. 6 were obtained from one spinal cat by simultaneous recordings of the excitatory and inhibitory EDRs. The right cutaneous nerve of the L1
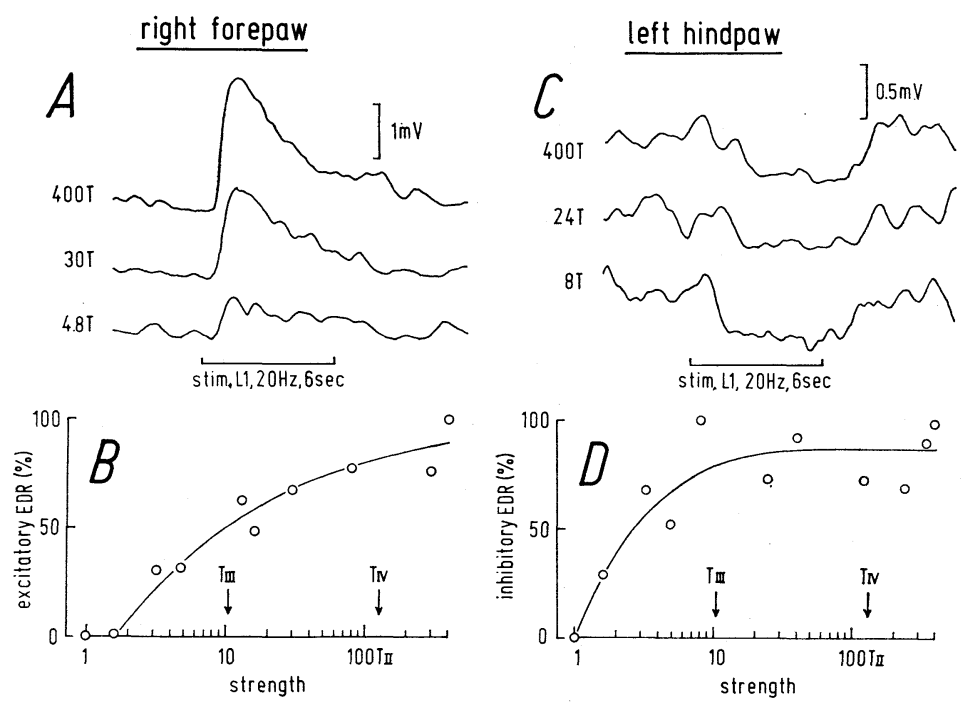

Fig. 6. Relations between EDR magnitude and intensity of electrical stimulation of cutaneous nerve. Excitatory and inhibitory EDRs recorded from right forepaw (A, B) and left hindpaw $(C, D)$. Right lateral cutaneous nerve branch of the L1 spinal segment was stimulated. Except for the stimulus intensity, other parameters were fixed: frequency, $20 \mathrm{~Hz}$; pulse duration, $0.5 \mathrm{msec}$; stimulus duration, $6 \mathrm{sec}$. Stimulus intensities were expressed as multiples of threshold intensities for group II fibers. A and C, specimen records of excitatory and inhibitory EDRs; B and D, relations between intensity of cutaneous stimulation (abscissae) and magnitudes of excitatory EDR (B) and inhibitory EDR (D) (ordinates). The threshold intensities for the group III and IV fibers are indicated by arrows in the graphs. 
segment was stimulated for $6 \mathrm{sec}$ with varying intensities. A and $\mathrm{C}$ show specimen records for the averaged excitatory and inhibitory EDRs, respectively, evoked by three different stimulus intensities. The stimulus intensities were expressed as multiples of the threshold for the group II fibers.

Figure $6 \mathrm{~B}$ and $\mathrm{D}$ show graphically the relations between stimulus intensity (abscissae) and response magnitude (ordinates); $\mathrm{B}$ for the excitatory EDR and D for the inhibitory EDR. For these graphs the response magnitudes were measured in the following way. First, the baseline was estimated from the deflections of SPWs for a pre-stimulus period of $4 \mathrm{sec}$. Then, the area delimited by this baseline and the response curve was measured with a planimeter for a period of $7.5 \mathrm{sec}$ starting $0.5 \mathrm{sec}$ after the onset of stimulation (The deflection of EDR, either excitatory or inhibitory, usually returned to the estimated baseline within $10 \mathrm{sec}$ ). These response areas were then converted into percentages of the largest excitatory and inhibitory EDRs. Both excitatory and inhibitory EDRs were produced by stimulation of group II fibers. However, some difference was found between the two kinds of EDR in their reaction to increase of the stimulus strength; the excitatory EDR continued to increase with increases in stimulus intensity beyond the threshold intensity for the group IV fibers, while the inhibitory EDR reached its maximum before the stimulus intensity reached the threshold for the group III fibers. Results similar to those of Fig. 6 were confirmed in 4 other cats.

\section{DISCUSSION}

The present experiment demonstrated that, in spinal cats pinching the skin or stimulating cutaneous afferent nerves at various spinal segments produced excitatory or inhibitory EDRs. Although the excitatory EDR has been well documented (see WANG, 1964), this paper is the first report of inhibitory EDRs. As both the inhibitory and excitatory EDRs disappeared after i.v. administration of atropine or after denervation of the sudomotor nerves, they would seem to be produced by changes in sudomotor activity at the spinal level. It is considered especially interesting to find both excitatory and inhibitory neural reflex mechanisms at the spinal level for the autonomic nervous system.

Whether the EDR was excitatory or inhibitory, was largely determined by the level of the spinal segmental afferent stimulated. For example, the inhibitory EDRs of the hindpaw were more frequently produced by stimulation of afferent nerves of the caudal thoracic and rostral lumbar segments, whereas excitatory EDRs of the hindpaw were elicited more frequently from mid-thoracic nerves. Afferent nerves which were effective in eliciting one type of EDR were relatively or completely ineffective for eliciting the other type of EDR. It is known that the sudomotor fibers innervating the hindpaw derive from the caudal thoracic and rostral lumbar spinal segments and those innervating the forepaw derive from the rostral thoracic segments (LANGLeY, 1891, 1894-1895; Patton, 1948). It is 
also known that the cutaneous afferents in cats are arranged in a segmental manner (KuHn, 1953; HeKmatPanah, 1961) which corresponds to Head's zones found in humans. Thus, our present results can be explained as follows: stimulation of cutaneous afferent nerves entering the spinal cord in the same segments as the sudomotor outflow or proximately to them will inhibit sudomotor activity. Conversely, stimulation of cutaneous afferent nerves entering the spinal cord at segments distal from the sudomotor outflow will excite sudomotor activity.

In the present experiments, inhibitory EDRs in one paw were more frequently produced by ipsilateral than by contralateral cutaneous stimulation. It is conceivable that this phenomenon could result from a mechanism similar to the one which produces such hemihidrosis in human beings as found by TAKAGI and SAKURAI with pressure stimulation upon the body surface (1950). Hemihidrosis is a condition in which there is excessive perspiration contralateral to the side pressed and depressed perspiration ipsilateral to the side pressed. However, in Takagi and Sakurai's experiments pressure stimuli might have been delivered not only to the skin, but also to the muscle. It might be of interest to examine whether the hemihidrosis reported by Takagi and Sakurai could be elicited by pinching the skin only, not stimulating the muscle. Additionally, it would be worthwhile examining the possibility of eliciting inhibitory EDRs by pressure stimulation of muscles.

KARL et al. (1975) found that electrical stimulation of only the group II cutaneous afferents produced a maximal excitatory EDR in chloralose anesthetized cats whose central nervous system was intact. No inhibitory EDRs were reported. Our present results demonstrate the contribution of the group III and IV cutaneous afferents to the excitatory EDR in spinal cats in addition to the group II afferents. This suggests that in the experiments of KARL et al. (1975) the contributions of the group III and IV afferents to EDR excitation were either minimal or absent due to inhibition by either the supraspinal structures or chloralose anesthesia. One reason why inhibition was not seen in the experiments of KARL et al. (1975) was that only limb afferents were stimulated.

As noted in RESULTS, there were several instances where the EDRs were not uniform in the amount of response or were inconsistent in the response type. For example, stimulation of the same nerve or skin area produced EDRs which were sometimes excitatory and at other times inhibitory. At the time of writing we are not sure what factors are responsible for these inconsistencies. However, based on preliminary observations, we suspect that the concentration of $\mathrm{O}_{2}$ and $\mathrm{CO}_{2}$ in the blood and spinal cord are contributory. Investigations are continuing to determine possible additional factors.

The authors express their gratitude to Professors A. Simpson and Jon Ek for reading and criticizing the manuscript. This work was supported by a research grant (No 257038) from the Ministry of Education, Science and Culture of Japan. 


\section{REFERENCES}

GILDEMEISTER, M. (1928) Der galvanische Hautreflex. In: Handbuch der Normalen und pathologischen Physiologie, ed. by Bethe, A., Bergmann, G. V., Embden, G., and Ellinger, A. Springer, Berlin, Vol. 8, Part 2, pp. 657-1095.

HekmatpanaH, J. (1961) Organization of tactile dermatomes, C1 through L4, in cat. $J$. Neurophysiol., 24: 129-140.

KARL, H., SATo, A., and Schmidt, R. F. (1975) Electrodermal reflexes induced by activity in somatic afferent fibers. Brain Res., 87: 145-150.

KuHN, R. A. (1953) Organization of tactile dermatomes in cat and monkey. J. Neurophysiol., 16: 169-182.

LADPLI, R. and WANG, G. H. (1960) Spontaneous variations of skin potentials in footpads of normal, striatal and spinal cats. J. Neurophysiol., 23: 448-452.

LANGLEY, J. N. (1891) On the course and connections of the secretory fibers supplying the sweat glands of the feet of the cat. J. Physiol. (Lond.), 12: 347-374.

LANGLEY, J. N. (1894-1895) Further observations on the secretory and vasomotor fibers of the foot of the cat. J. Physiol. (Lond.), 17: 296-314.

Patton, H. D. (1948) Secretory innervation of the cat's food-pad. J. Neurophysiol., 11:211227.

TAKAGI, K. and SAKuraI, T. (1950) A sweat reflex due to pressure on the body surface. Jap. J. Physiol., 1: 22-28.

WANG, G. H. (1957) The galvanic skin reflex. A review of old and recent works from a physiologic point of view. Part 1. Am. J. Phys. Med., 36: 295-320.

WANG, G. H. (1958) The galvanic skin reflex. A review of old and recent works from a physiologic point of view. Part 2. Am. J. Phys. Med., 37: 35-57.

WANG, G. H. (1964) The Neural Control of Sweating, Univ. Wisconsin Press, Madison.

Vol. 28 , No. 6,1978 